\title{
Evaluación de los factores estratégicos del sector hotelero en la provincia de Sabana Centro, Colombia
}

Evaluation of the strategic factors of the hotel sector in the province of Sabana Centro, Colombia

Elka Liliana Arias-Olmos (iD) Universidad de La Sabana, Colombia elkaarol@unisabana.edu.co

Maricela Isabel Montes-Guerra (iD) Universidad de La Sabana, Colombia maricela.montes@unisabana.edu.co

Guido Angello Castro-Ríos (iD, Universidad de La Sabana, Colombia guido.castro@unisabana.edu.co

\section{RESUMEN}

El artículo busca identificar la presencia de factores estratégicos internos en los establecimientos de alojamientos y hospedaje de la provincia de Sabana Centro en el departamento de Cundinamarca, Colombia. Se realizó el análisis a partir de fundamentos teóricos de tres factores estratégicos internos que soportan la creación de ventajas competitivas a largo plazo: activos organizacionales, marketing mix y activos reputacionales. Para la recolección de la información se desarrolló un instrumento que se aplicó a los establecimientos formalmente constituidos en la zona. Los resultados evidencian que en su mayoría los establecimientos de alojamiento y hospedaje en la provincia de Sabana Centro adolecen de los factores estratégico internos sugeridos por la teoría de management. Podrían ser apoyados por instituciones públicas y privadas para implementarlos y así impulsarlos a tener una oferta más consistente y competitiva. Esta investigación finalizó meses antes del inicio la pandemia provocada por la COVID-19 y sus efectos no fueron estudiados, pero los resultados aportan a su recuperación en un futuro próximo.

Palabras clave: Competitividad; desempeño; industria del turismo; hospedaje. 


\section{ABSTRACT}

This article seeks to identify the presence of internal strategic factors in accommodation and lodging establishments in the province of Sabana Centro in the department of Cundinamarca, Colombia. The analysis was carried out based on the theoretical foundations of three internal strategic factors that support the creation of long-term competitive advantages: organizational assets, marketing mix and reputational assets. To collect the information, an instrument was developed that was applied to the establishments formally established in the area. The results show that most of the accommodation and lodging establishments in the province of Sabana Centro suffer from the internal strategy factors suggested by management theory. They could be supported by public and private institutions to implement them and thus encourage them to have a more consistent and competitive offer. This research ended months before the start of the pandemic caused by COVID-19 and its effects were not studied, but the results can contribute to its recovery in the near future.

Keywords: Competitiveness; performance; tourism industry; lodging.

\section{INTRODUCCIÓN}

El turismo es una industria dinámica cuyo funcionamiento depende de los recursos que la conforman y las capacidades para gestionarlos. Tal es el caso de los establecimientos de alojamiento y hospedaje (EAH) donde, usualmente, su base se concentra en las edificaciones en las cuales se presta el servicio y se complementa con las capacidades para gestionar personas, procesos, proveedores, clientes, comunicaciones, entre otras.

La Cámara de Comercio de Bogotá realizó en el año 2005 un estudio que permitió caracterizar las cadenas productivas de manufactura y servicios en la ciudad de Bogotá y el departamento de Cundinamarca, dicho estudio afirma que la conformación y gestión de cadenas productivas promueven la competitividad y la productividad de la región mencionada. En esta caracterización se indica que en la cadena productiva correspondiente al sector turístico el eslabón que contiene la mayor concentración de actores es el de prestación del servicio de alojamiento. Este eslabón está conformado por establecimientos tales como hoteles, hostales, apartahoteles, entre otros (Cámara de Comercio de Bogotá, 2005).

En Cundinamarca los hoteles, restaurantes y bares realizan un aporte importante a su economía, con una participación del 15,4\% en el PIB departamental después de la industria manufacturera la cual representa el 20,9\% (MinCIT, 2018). Entre los años 2000 y 2016 la participación promedio de hoteles, restaurantes y bares en el PIB de Cundinamarca fue de $3,1 \%$ y aumentó al 3,6\% en el 2016. Según cifras del Centro de Información Turística de Colombia (CITUR), en el año 2019 Colombia registró 27.540 EAH, en donde el departamento de Cundinamarca (sin incluir Bogotá) representa el 6,17\% de la oferta nacional de EAH con Registro Nacional de Turismo (MinCIT-CITUR, 2019).

Respecto a la situación del sector turismo en la zona de estudio, no se identificó ningún diagnóstico sobre los EAH que permita mejorar la inversión público-privada y definir estrategias para potencializar este actor estratégico del sector turístico. El presente estudio se enfoca en los proveedores de alojamiento por su papel en la especialización de los espacios convertidos en destinos turísticos, dado que son una fuente de empleo estable, realizan la 
legalización de sus instalaciones, hacen contribuciones fiscales y generan rentas (Pozo, Campos, Sánchez y Lara, 2011). En adición, estos actores contribuyen a que el cliente perciba un alto valor de experiencia en el destino (Brathwaite, 1992) ya que en la cadena de valor del turismo intervienen en la fase de estadía en sitio (Varvaressos, 2018) que es donde el viajero suele pasar mayor tiempo. Estos establecimientos suelen adquirir un rol de catalizador del desarrollo económico y social en una zona o región particular donde el turismo se ha seleccionado como un sector estratégico.

Los objetivos de esta investigación se centran en caracterizar la oferta de EAH presentes en la provincia Sabana Centro en el departamento de Cundinamarca, Colombia, y determinar la presencia de los factores estratégicos internos de los EAH requeridos para que éstos logren desempeñarse mejor que su competencia y puedan establecer diferencias que sean valoradas por sus huéspedes. En este estudio se analizan los factores propuestos por González-Rodríguez, Jiménez-Caballero, Martín-Samper, Köseoglu, y Okumus (2018): activos organizacionales y activos reputacionales, y se complementan con el concepto de marketing mix, con lo cual se plantean tres factores estratégicos internos necesarios para un mejor desempeño y competitividad.

Entre los principales hallazgos cabe mencionar que la oferta de EAH existente en la región es disímil y permanece a la espera de un mercado natural sujeto a una alta variabilidad, no existe una diferenciación clara entre los establecimientos presentes en la región que se enfoque en atraer variedad de huéspedes y genere mayor fidelidad entre los mismos. De igual manera, se identificaron brechas en los factores estratégicos internos necesarios para que los EAH de la provincia Sabana Centro logren desarrollar ventajas competitivas sostenibles, especialmente los establecimientos de menor tamaño.

Los resultados de esta investigación constituyen un marco referencial que contribuye a la preparación de planes de desarrollo turístico en la región, sirve para proponer esquemas de apoyo para el crecimiento de la industria e identificar las oportunidades que reduzcan las brechas en los factores de estrategia aplicados por los EAH y que son determinantes para que los mismos obtengan una mejor competitividad, teniendo en cuenta que a largo plazo el éxito empresarial se sustenta en la gestión adecuada de los recursos y la creación de capacidades organizacionales.

\section{DESCRIPCIÓN DE LA ZONA ESTUDIADA}

La zona seleccionada para el estudio corresponde a la provincia Sabana Centro, ubicada en el departamento de Cundinamarca, Colombia (figura 1), la cual está compuesta por 11 municipios (municipalidades): Cajicá, Chía, Cogua, Cota, Gachancipá, Nemocón, Sopó, Tabio, Tenjo, Tocancipá y Zipaquirá. Esta provincia es una de las más atractivas del departamento debido a la riqueza histórica, cultural, natural y a su ubicación geográfica cercana a la ciudad de Bogotá (Sabana Centro Cómo Vamos, 2016). La provincia de Sabana Centro se entiende como el socio natural para posicionar a la capital del país como un destino turístico atractivo a nivel nacional e internacional (Consejo Regional de Competitividad, 2010). En la tabla 1 se presenta información sobre el tamaño de la población en cada municipio y algunos de sus atractivos turísticos. 
Figura 1. Ubicación de la provincia Sabana Centro en el departamento de Cundinamarca, Colombia

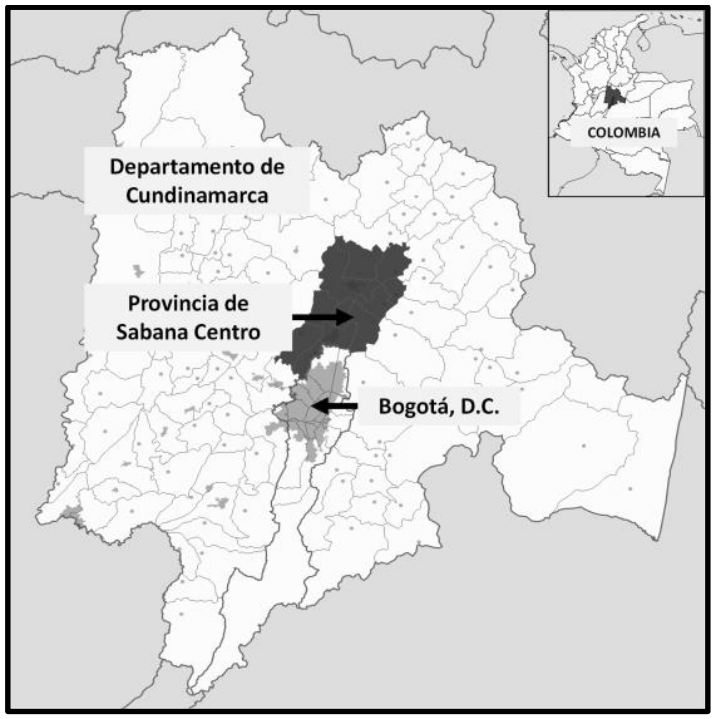

Elaborado sobre imagen disponible en Wikipedia: https://commons. wikimedia.org/w/index.php?curid=45656362

Tabla 1. Información de los municipios y atractivos de la Provincia

\begin{tabular}{|c|c|c|}
\hline Municipio & Población & Atractivo turístico \\
\hline Zipaquirá & 130,537 & La Catedral de Sal- Parque de la Sal \\
\hline Chía & 132,181 & $\begin{array}{l}\text { Puente del Común, Restaurante Andrés Carne de Res, Castillo } \\
\text { Marroquín, Festival Estéreo Picnic }\end{array}$ \\
\hline Tocancipá & 39,996 & $\begin{array}{l}\text { Autódromo de Tocancipá, Iglesia de los Caballeros de la Virgen, Parque } \\
\text { Jaime Duque }\end{array}$ \\
\hline Sopó & 25,782 & $\begin{array}{l}\text { Parke Finkana, Cabaña de Alpina, Cerro Pionono, Santuario Jesús de la } \\
\text { Piedra }\end{array}$ \\
\hline Cajicá & 82,244 & $\begin{array}{l}\text { Parque de la estación del tren, Parroquia Inmaculada de la Concepción, } \\
\text { Centro Cultural, Periland Eco Parque, Camping Cajicá }\end{array}$ \\
\hline Gachancipá & 17,026 & $\begin{array}{l}\text { Iglesia de San Bartolomé, Observatorio El Caolín, Cueva de los } \\
\text { murciélagos }\end{array}$ \\
\hline Tabio & 21.665 & Capilla de Santa Bárbara, Termales del Zipa, Peña de Juaica \\
\hline Cogua & 22,067 & $\begin{array}{l}\text { Represa del Neusa, Foyer de Charité, Vereda la Plazuela, pictogramas } \\
\text { Muiscas en la Vereda Patasica }\end{array}$ \\
\hline Cota & 32,691 & $\begin{array}{l}\text { Hacienda el Noviciado, Pictograma Piedra de la Tapia, tejidos en lana, } \\
\text { Pintura en tela y muñequería }\end{array}$ \\
\hline Tenjo & 21,935 & $\begin{array}{l}\text { Sendero Ecoturístico las Cuevas, restos del Santuario Muisca, Reserva } \\
\text { de orquídeas }\end{array}$ \\
\hline Nemocón & 13,171 & $\begin{array}{l}\text { Mina de sal de Nemocón, Museo de la sal o casa del Encomendero, } \\
\text { Teatro Municipal }\end{array}$ \\
\hline
\end{tabular}

El dinamismo del sector turismo en Cundinamarca se evidencia por el crecimiento de los prestadores de servicios turísticos activos en el Registro Nacional de Turismo (RNT), los cuales entre el año 2013 y el año 2020 aumentaron un 73\% en total (tabla 2). 
Tabla 2. Indicadores sectoriales para EAH ubicados en Cundinamarca

\begin{tabular}{lrrrrrrrr}
\hline Indicador & $\mathbf{2 0 1 3}$ & $\mathbf{2 0 1 4}$ & $\mathbf{2 0 1 5}$ & $\mathbf{2 0 1 6}$ & $\mathbf{2 0 1 7}$ & $\mathbf{2 0 1 8}$ & $\mathbf{2 0 1 9}$ & $\mathbf{2 0 2 0}$ \\
& & & & & & & & \\
\hline $\begin{array}{l}\text { Prestadores de servicios } \\
\text { turísticos activos RNT }\end{array}$ & 705 & 868 & 963 & 1.206 & 1.372 & 1.522 & 2.404 & 2.655 \\
\hline $\begin{array}{l}\text { Establecimientos de } \\
\text { alojamiento y hospedaje }\end{array}$ & 450 & 579 & 662 & 801 & 954 & 1.057 & 1.700 & 1.986 \\
\hline Ocupación hotelera (\%) & 34,10 & 35,70 & 37,10 & 37,75 & 32,80 & ND & ND & ND \\
\hline Total habitaciones EAH & 10.492 & 12.435 & 13.461 & 13.547 & 15.318 & 44.807 & 21.011 & 22.605 \\
& & & & & & & & \\
\hline Total camas EAH & 22.534 & 25.531 & 27.128 & 26.086 & 29.482 & 73.199 & 39.655 & 41.495
\end{tabular}

Fuente: MinCIT-CITUR (2021)

De acuerdo con los planes de desarrollo del departamento de Cundinamarca 20162020 y 2020-2024, el turismo es una de las actividades económicas que puede aportar a la competitividad departamental, es un instrumento para luchar contra la pobreza y es una herramienta primordial para el desarrollo. Sin embargo, se menciona que el departamento afronta dificultades de infraestructura, accesibilidad a los atractivos y una deficiente calidad en la prestación de los servicios turísticos (Gobernación de Cundinamarca, 2016). En el plan de desarrollo vigente (2020-2024), se han planteado como retos el diseño de productos turísticos, la acreditación de destinos sostenibles y la creación de un observatorio de turismo para la toma de decisiones inteligentes (Gobernación de Cundinamarca, 2020) entre otras acciones para fortalecer el sector en el largo plazo.

\section{FACTORES ESTRATÉGICOS INTERNOS}

Uno de los planteamientos sobre estrategia con aplicación a todo tipo de industria está dado por Porter (1996), quien afirma que las empresas se pueden desempeñar mejor que sus rivales sólo si son capaces de establecer una diferencia que puedan mantener. También considera la estrategia como un sistema de actividades donde las estructuras, los procesos y los sistemas de una organización se encuentran articulados. Enríquez De La O (2015) afirma que "Los modelos de gestión han definido tradicionalmente crear un valor único a través de estrategias basadas en producto o posicionamiento de mercado" (2015, p. 51). En el caso de los EAH, el análisis de sus recursos y capacidades cobran especial importancia en el desarrollo de su estrategia, dado que proporcionan una ventaja competitiva más duradera que la visión sustentada en la relación producto/mercado.

Respecto a las capacidades de una empresa, Amit y Schoemaker (1993) consideran que son la forma en la cual la empresa combina sus recursos. Además, plantean el concepto de Factores Críticos de Éxito con las nociones de: (a) factores estratégicos de la industria, conjunto de recursos y capacidades que son determinantes para las ganancias de los participantes de la industria y (b) activos estratégicos como el conjunto de recursos y capacidades desarrolladas por la gerencia para crear y proteger a la ventaja competitiva de la empresa. Para diferenciarse de la competencia es necesario que los recursos y las capacidades, incorporados en la estrategia sean heterogéneas y no puedan ser transferibles de una empresa a otra (González-Rodríguez et al., 2018; Köseoglu, Altin, Chan, Aladag, 2020). De manera empírica Rubio y Aragón (2008) afirman que el éxito de las pequeñas y medianas 
empresas, como es el caso de los EAH abordados en este estudio, dependen de recursos y capacidades similares a los que tienen grandes empresas.

Las empresas de hotelería y turismo deben identificar los recursos y las capacidades necesarias para crear ventaja competitiva. Gursoy y Swanger (2007) examinaron los factores estratégicos internos que determinan el desempeño financiero de este tipo de organizaciones, entre ellos se destacan: esfuerzos en ventas, investigación y desarrollo, distribución, tecnología de información y recursos Humanos. Por otro lado, Langvinienè y Daunoravičiūtè (2015) plantean como factores de éxito en el negocio de la hospitalidad: el mercadeo interno, la propuesta de valor, la gerencia de las relaciones con los clientes, el empoderamiento de los empleados, la tecnología y la innovación. Adicional a lo anterior, en la industria del turismo y la hospitalidad es relevante el factor reputacional, especialmente en las redes sociales para turistas, dado que son fuente de influencia para los clientes potenciales en términos de percepción de fiabilidad, calidad y lo que se está dispuesto a pagar (Bačík et al., 2019).

Por su parte, García (2013) menciona que los activos intangibles son impulsores clave del proceso de creación de valor y contribuyen al logro de ventajas competitivas. El autor hace referencia a la incorporación de intangibles en los procesos internos clave de la empresa, tales como: Conocimientos, capacidades, recursos organizativos, de información e innovación y al despliegue de redes de relaciones con el exterior. Entendiendo los postulados anteriores, se toma como referencia el estudio de González- Rodríguez et al. (2018) en el cual identifican los factores de estrategia que impulsan el desempeño de los hoteles, siendo estos: (a) activos organizacionales donde plantean experiencia gerencial, clima organizacional, coordinación en la organización, entre otros, (b) capacidades de mercadeo donde debe tenerse control y acceso a canales, el conocimiento del mercado, una sólida base de clientes, entre otros, (c) activos reputacionales con respecto al servicio al cliente y (d) capacidades técnicas donde evalúan recursos tecnológicos tales como centrales de reservas, equipos del hotel y localización.

En la tabla 3 se resumen los planteamientos sobre factores estratégicos internos que plantean González- Rodríguez et al. (2018) y se complementan con el concepto de marketing mix, con lo cual se proponen tres factores estratégicos relevantes para el éxito de un EAH: activos organizacionales, marketing mix y, activos reputacionales. 
Tabla 3. Factores internos que impactan la estrategia de los EAH

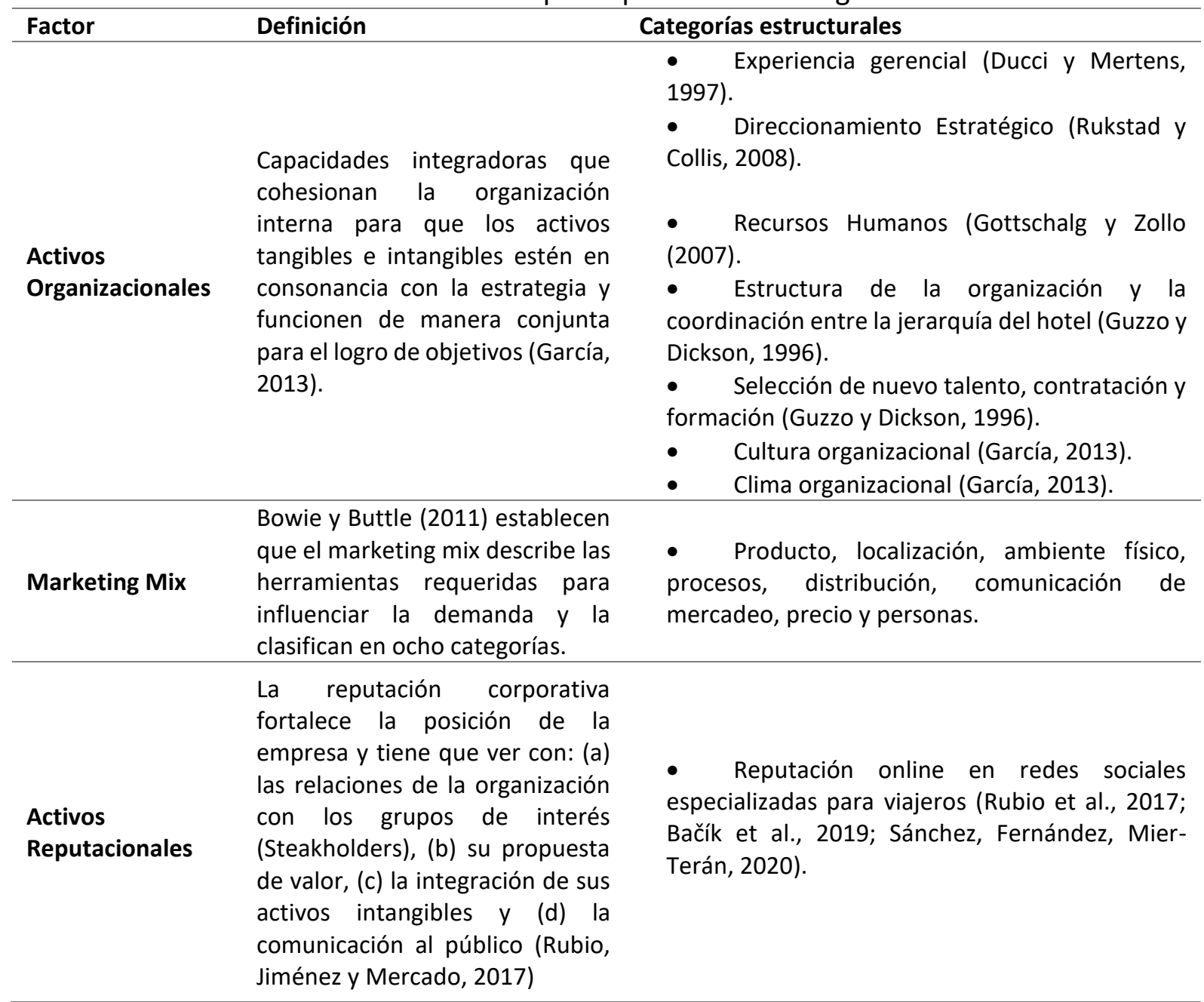

Elaboración propia

En todo caso, los factores estratégicos internos tienen un carácter dinámico, es decir, requieren de una continua renovación de competencias para lograr congruencia con los cambios del entorno de negocios y reconfigurarse en sus habilidades organizacionales internas y externas, para coincidir con los requerimientos de un ambiente cambiante (Wernerlfet, 1984). Luego, es posible afirmar que la estrategia de acumular recursos a menudo no es suficiente para soportar una ventaja competitiva en esta industria.

Autores como Camisón et al., (2015) han realizado estudios para establecer los efectos de los recursos, las capacidades y la localización de la organización turística sobre su posición competitiva. Sus resultados permiten explicar la competitividad en las empresas turísticas a partir de: (a) la gerencia de los recursos y las capacidades, (b) la forma como individualmente las empresas turísticas presentes en un distrito aprovechan los beneficios por pertenecer a él, superando esto al argumento tradicional que afirma que la ubicación es el factor más importante para lograr competitividad, y (c) el turismo como actividad económica en la que no se venden recursos físicos y, por lo tanto, éstos no tienen una influencia significativa en la competitividad.

En las empresas turísticas los mercados son de alta competencia y los entornos pueden cambiar en cuestión de horas (Ej.: terrorismo, seguridad del país, pandemias). Los directivos 
de estas organizaciones deben ser conscientes que la competitividad se genera respecto a factores como la capacidad de creación, la marca (Foroudi, 2020), la transferencia de conocimiento, la gestión adecuada del recurso humano, la cultura organizacional, la cohesión de los equipos, la óptima coordinación, el empeño por proporcionar servicios de calidad y la comercialización integrada al destino turístico e innovación. Es importante, además, el aporte de los recursos físicos y financieros en la construcción de una ventaja competitiva, ya que una base financiera sólida es esencial para asumir inversiones en intangibles con rendimientos a largo plazo.

\section{METODOLOGÍA}

El diseño en este estudio es de tipo mixto y concurrente, dado que la información cualitativa y cuantitativa se recopiló de manera simultánea (Sampieri y Torres, 2018). Esta recopilación se efectuó a través de la aplicación de encuestas y mediante observación in situ a los EAH. La población entrevistada se concentró en gerentes, administradores y/o propietarios de los EAH pertenecientes a la provincia Sabana Centro.

Se identificaron 69 EAH registrados en el RNT para el año 2019 (figura 2). Cabe aclarar, que la muestra incluye las clasificaciones de establecimientos de alojamiento y hospedaje (EAH) bajo el concepto de la norma técnica sectorial colombiana NTSH 006 (MinCIT, 2009), la cual incluye a los siguientes establecimientos bajo la categoría de EAH: apartahotel, centro vacacional, hotel, hostal, alojamiento rural y otros tipos (donde se incluyen apartamentos turísticos y posadas turísticas). La encuesta se realizó $55 \mathrm{EAH}$, los restantes no fueron contemplados porque no se encontró al administrador o propietario, manifestaron que no les interesaba participar o el establecimiento estaba cerrado.

Figura 2. Distribución de EAH en los municipios de la provincia de Sabana Centro

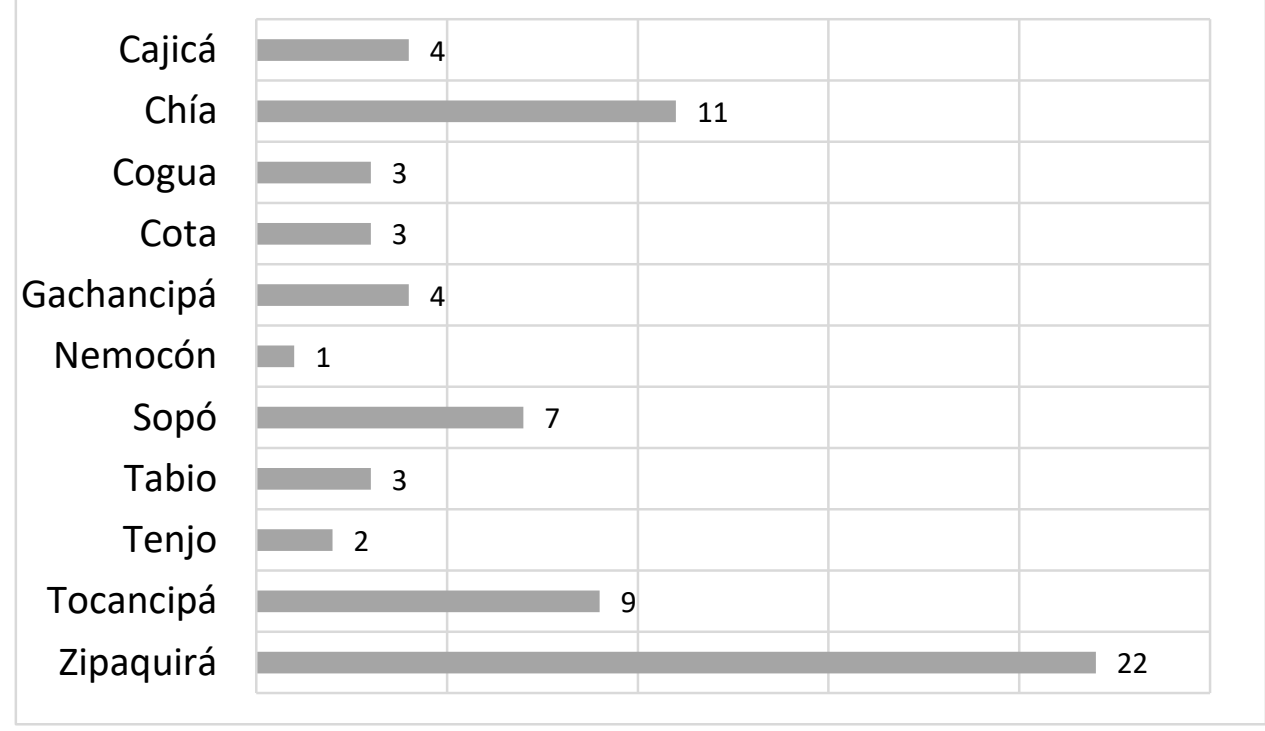

Elaboración propia 


\subsection{Instrumento y técnicas de recolección de datos}

Con el fin de evidenciar la presencia de factores estratégicos internos se diseñó un instrumento tipo encuesta elaborado a partir del conocimiento de la industria y de la revisión de diferentes propuestas. El cuestionario está dividido en cinco secciones con un total de 74 preguntas, como se explica en la tabla 4. El cuestionario se basa en los trabajos de Bowie y Buttle (2011), García (2013), González et al. (2018), Millán y Gómez (2018) y Scheel (2010). El instrumento fue revisado por cinco expertos, dos de ellos académicos y tres de la industria hotelera, quienes sugirieron ajustes para facilitar la comprensión de algunos ítems. Posteriormente se realizó una prueba piloto a un grupo de nueve gerentes de hoteles de una zona diferente a la del estudio.

Tabla 4. Descripción de la estructura del instrumento de medición y fuentes consultadas

\begin{tabular}{|c|c|c|c|c|}
\hline Sección & $\begin{array}{l}\text { Denominación de la } \\
\text { sección }\end{array}$ & $\begin{array}{l}\text { Número de } \\
\text { preguntas }\end{array}$ & Descripción del contenido & Fuentes \\
\hline 1 & $\begin{array}{l}\text { Caracterización del } \\
\text { encuestado }\end{array}$ & 10 & $\begin{array}{l}\text { Información del gerente, } \\
\text { administrador o propietario } \\
\text { que se encuentra } \\
\text { respondiendo la encuesta. }\end{array}$ & No aplica \\
\hline 2 & $\begin{array}{l}\text { Caracterización del } \\
\text { EAH }\end{array}$ & 13 & $\begin{array}{l}\text { Información específica del } \\
\text { EAH. }\end{array}$ & No aplica \\
\hline \multirow{3}{*}{3} & $\begin{array}{l}\text { Factores estratégicos: } \\
\text { Activos } \\
\text { Organizacionales }\end{array}$ & 11 & $\begin{array}{l}\text { Información sobre el primer } \\
\text { factor estratégico - activos } \\
\text { organizacionales el cual se } \\
\text { dividen en: 1) } \\
\text { Direccionamiento Estratégico } \\
\text { y 2) Recursos Humanos. }\end{array}$ & $\begin{array}{l}\text { García(2013) } \\
\text { González, et al (2018) } \\
\text { Millán y Gómez (2018) }\end{array}$ \\
\hline & $\begin{array}{l}\text { Factores estratégicos: } \\
\text { Marketing Mix }\end{array}$ & 24 & $\begin{array}{l}\text { El segundo factor estratégico } \\
\text { analizado es el marketing mix, } \\
\text { los cuales corresponden a: } \\
\text { Localización, ambiente físico, } \\
\text { procesos, distribución, } \\
\text { comunicación, precio y gente. }\end{array}$ & $\begin{array}{l}\text { Bowie y Buttle (2011) } \\
\text { García (2013) } \\
\text { González, et al (2018) } \\
\text { Millán y Gómez (2018) }\end{array}$ \\
\hline & $\begin{array}{l}\text { Factores estratégicos: } \\
\text { Activos } \\
\text { Reputacionales }\end{array}$ & 5 & $\begin{array}{l}\text { El tercer factor estratégico } \\
\text { indagado fue el activo } \\
\text { reputacional (on-line). }\end{array}$ & $\begin{array}{l}\text { García (2013) } \\
\text { González, et al (2018) } \\
\text { Millán y Gómez (2018) } \\
\text { Rubio, et al (2017) }\end{array}$ \\
\hline 4 & $\begin{array}{l}\text { Posición en el } \\
\text { mercado }\end{array}$ & 3 & $\begin{array}{l}\text { Determinar el conocimiento } \\
\text { del EAH sobre la competencia } \\
\text { y el entendimiento de su } \\
\text { posición con respecto a ella. }\end{array}$ & González, et al (2018) \\
\hline 5 & $\begin{array}{l}\text { Fortalezas para } \\
\text { competir }\end{array}$ & 8 & $\begin{array}{l}\text { A partir de los elementos del } \\
\text { marketing mix se formulan } \\
\text { preguntas para entender la } \\
\text { percepción del EAH con } \\
\text { respecto a los elementos en } \\
\text { los cuales es fuerte para } \\
\text { competir. }\end{array}$ & González, et al (2018) \\
\hline
\end{tabular}

Elaboración propia

\subsection{Técnica de análisis estadístico de información}

Con relación a las variables cualitativas de los datos recolectados de los EAH, se realizó un análisis descriptivo de la información obtenida. Para el análisis cuantitativo se trabajó bajo 
el marco de un diseño factorial y análisis de correspondencia múltiple (ACM) el cual permite manipular dos o más variables independientes e incluyen dos o más modalidades de presencia en cada una de las variables independientes (Sampieri y Torres, 2018, p. 148). Por tanto, el análisis se hizo a través de un método de interdependencia que busca el cómo y el por qué se relacionan o asocian un conjunto de variables (Díaz y Morales, 2012, p. 15). La técnica de estadística descriptiva específica que se utilizó fue un análisis de correspondencia que tiene como objetivo representar las tablas en las cuales se registran las frecuencias de aparición de dos o más variables cualitativas en un conjunto de elementos.

\section{RESULTADOS}

\subsection{Caracterización de la oferta de EAH en la Provincia Sabana Centro}

En la provincia Sabana Centro, el $67 \%$ de los EAH están clasificados como hoteles y concentran el $89 \%$ del total de las habitaciones, el $85 \%$ de los hoteles se encuentra en un rango de 2 a 39 habitaciones y la oferta restante va de 64 a 128 habitaciones, de ellos el 69\% cuenta con menos de cuatro empleados que generalmente son familiares del propietario. Por otro lado, sólo en el $29 \%$ de los establecimientos quienes los dirigen cuentan con formación en hotelería, y solo el $47 \%$ manifiestan tener experiencia previa en el ramo. Adicionalmente, se evidenció que el $95 \%$ de los EAH son gestionados por sus propietarios, es decir no se amparan en cadenas operadoras de mayor experiencia gerencial que gestionen la comercialización ni guíen sus procesos hoteleros (ver figura 3).

Figura 3. Caracterización de los EAH de Sabana Centro

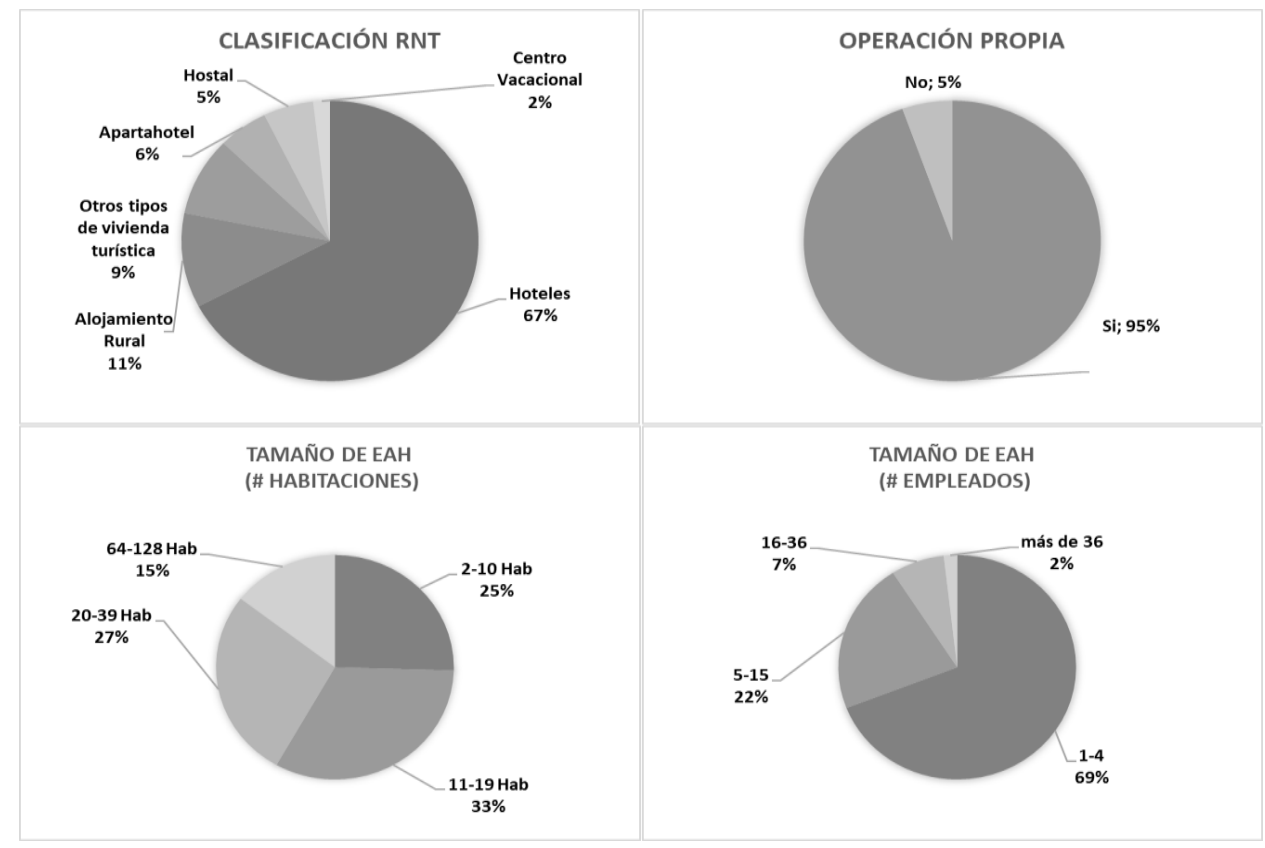

Elaboración propia

Cabe resaltar que a partir de la inspección visual y basados en el concepto dado por la normativa nacional, se encontró que el $19 \%$ de los EAH denominados como hotel no cuentan con los elementos para estar dentro de esta clasificación dado que no ofrecen desayuno, no tienen recepción ni el espacio común requerido para los huéspedes según la definición de la categorización por estrellas de la Norma Técnica Sectorial Hotelera NTSH 006 (MinCIT, 2009). 
En la provincia se destaca la presencia de los establecimientos: hotel GHL Style 80 y el hotel Factory Green en Cota, así como el hotel Clarión en Cajicá, los cuales cumplen con estándares internacionales y son operados por grupos hoteleros con experiencia, además ofertan más de 100 habitaciones. También se destaca las propuestas boutique, como La Posada del Ángel y el Camino de la Sal en Zipaquirá, que buscan atraer mercado internacional por su arquitectura tradicional y servicio. Es evidente que el surgimiento de los EAH en la provincia se ha dado en su mayoría por las oportunidades que han identificado en diferentes nichos como: transportadores, corporativos, deportistas, académicos, turistas y residentes de Bogotá. En los municipios más poblados se encontraron ofertas de alojamiento bajo RNT de hotel destinados a la prestación de servicio por hora principalmente para la población local.

\subsection{Presencia de factores estratégicos internos en los EAH de la Provincia Sabana Centro}

Se presentan los resultados de los hallazgos para los tres factores de estrategia analizados en este trabajo: activos organizacionales, marketing mix y activos reputacionales.

Activos organizacionales: Este factor evalúa las capacidades integradoras que cohesionan la organización interna para que los activos tangibles e intangibles estén en consonancia con la estrategia y funcionen de manera conjunta facilitando el logro de objetivos (García, 2013). En la figura 4 se muestran los hallazgos medidos sobre el porcentaje de establecimientos que tienen presencia de cada uno de los elementos que conforman los activos organizacionales.

Figura 4. Porcentaje de EAH con las variables analizadas en activos organizacionales

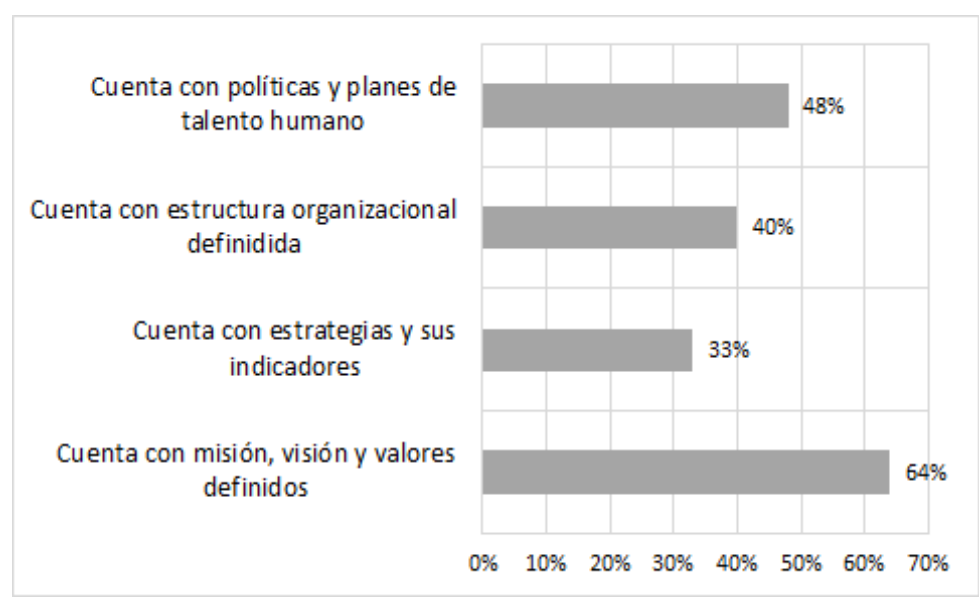

Elaboración propia

El 64\% de los EAH indican tener elementos de direccionamiento estratégico como lo son misión, visión y valores, pero solo el $33 \%$ de los EAH menciona que cuenta con estrategias para competir y sus respectivos indicadores. EL $40 \%$ ha definido una estructura organizacional y un $48 \%$ cuenta con políticas para la gestión del talento humano.

Marketing mix: Al momento de analizar las categorías referentes al factor de marketing mix se encontró que el $87 \%$ de los EAH afirma tener una buena localización para su mercado objetivo, el 95\% del producto de EAH de Sabana Centro cuenta con servicios básicos de agua caliente, amenidades de baño y televisión. Actualmente se encuentra la prestación del servicio de internet inalámbrico en el $89 \%$ de los establecimientos, pero solo el $45 \%$ de los EAH afirma contar con otros servicios complementarios tales como gimnasio, lavandería, parqueadero, 
minibar y/o spa. En los EAH no se evidenció en sitio la infraestructura para prestar servicios de alimentos y bebidas, siendo consistente con que sólo el $40 \%$ de los EAH brinda a los huéspedes algún servicio de restaurante, bar, servicio de alimentos a la habitación y/o salones de reuniones.

Sobre los procesos de conocimiento del cliente que utilizan los EAH, se encontró que el $55 \%$ de ellos realiza encuestas a los huéspedes para captar sus opiniones y, como herramienta de administración, sólo el $20 \%$ de los EAH cuenta con un software para la gestión de hoteles. En cuanto a las personas como base del servicio y conocimiento, solo el $31 \%$ de los EAH ha desarrollado protocolos de servicio que les permite asegurar la estandarización de la atención al huésped y el $58 \%$ de los EAH menciona que alguien de su equipo puede comunicarse en inglés y de ellos el $16 \%$ en otros idiomas. Mantener un adecuado ambiente físico de sus instalaciones es vital para este negocio y solamente el $42 \%$ de los EAH cuentan con planes de mantenimiento para su infraestructura, equipos y mobiliario.

Los canales de distribución de los EAH de la provincia son muy débiles: Solamente el $16 \%$ de los EAH ha estructurado un plan de ventas, el 29\% de los EAH está en agencias de viajes online y aunque el $60 \%$ de ellos afirma tener con una página web, no se evidencia que todas sean transaccionales. El complemento de venta es la comunicación del producto y sólo un $13 \%$ de los EAH menciona tener un plan de mercadeo. Por lo que se refiere a precios de venta se encontró que en general manejan tarifas fijas que pueden tener descuentos a discreción y el concepto de revenue management es implementado únicamente por el $16 \%$ de los EAH.

Activos reputacionales: Como factor estratégico interno se ha elegido la categoría de reputación online la cual se forma mediante la acumulación de juicios que emite el público que se relacionan con la empresa (Rubio et al., 2017) y a partir del análisis de dichos comentarios se puede identificar la satisfacción del cliente (Bačík et al., 2019) que puede traducirse en ventas y recompras. En este apartado se obtuvo que el $71 \%$ de los EAH afirma tener perfil en alguna red social como Facebook, Instagram o WhatsApp. Sin embargo, aun cuando los EAH menciona estar en redes sociales, se encuentra que solo un $55 \%$ de los EAH tiene comentarios en redes sociales de viajeros como Booking o Tripadvisor y de éstos el $23 \%$ tiene una calificación por debajo de 8.0 en una escala de 10 .

Fortalezas para competir: Para evaluar este factor se analizaron las percepciones de los EAH sobre las fortalezas que tienen con relación a sus competidores en temas de instalaciones, servicios disponibles, servicios de alimentación otros servicios complementarios y localización.

Con relación a estás fortalezas se analizó la frecuencia de categorías que se muestran en la figura 5 , se observa que un $87 \%$ de los EAH califican con muy débil y débil su capacidad para competir. Es importante indicar que estas percepciones recogen la información de los EAH que son operados por terceros, quienes tiene un promedio bajo al momento de calificar sus fortalezas para competir. Cabe resaltar que en la comparación que hacen los EAH sobre los servicios de alimentos un $22 \%$ manifiestan que cuenta con fortalezas para competir en este sector. 
Figura 5. Registro de frecuencia de fortalezas para competir de los EAH

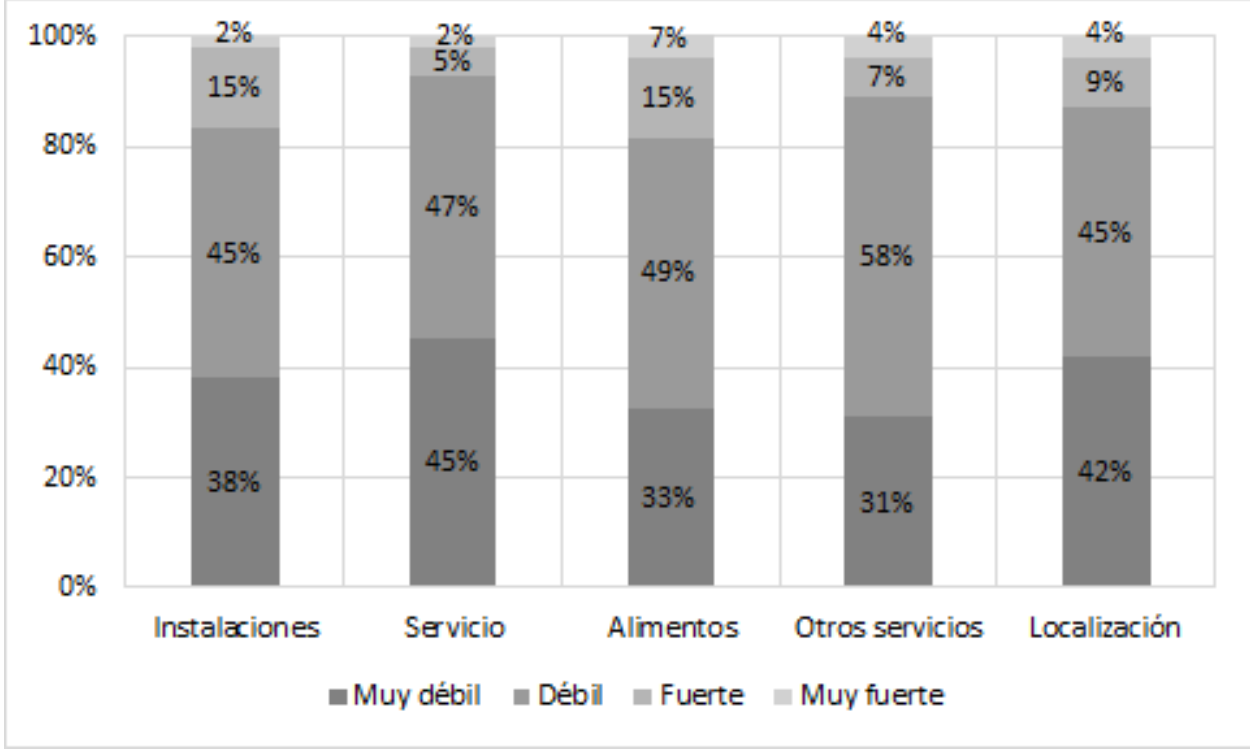

Elaboración propia

\subsection{Asociación entre factores estratégicos internos de los EAH}

Con el fin de disminuir el número de categorías de análisis, se agruparon los municipios que conforman la provincia en zonas utilizando los siguientes criterios: (a) municipios con características similares que permiten unirlos por su cercanía geográfica y el tipo de mercado que atienden, (b) número de EAH disponible en cada municipio. En la figura 6 se presentan las zonas en que se agruparon los EAH de la Provincia Sabana Centro.

Figura 6. Agrupamiento de las zonas de los EAH para análisis de ACM

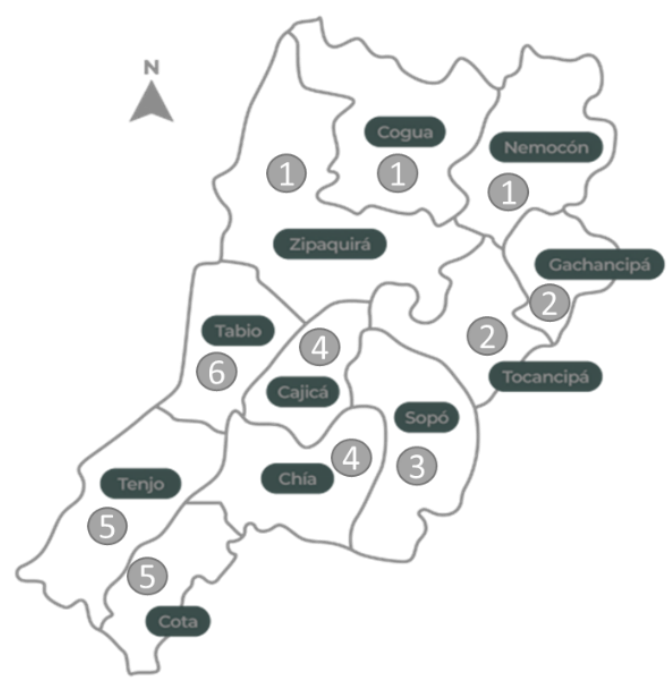

\begin{tabular}{|c|c|c|c|}
\hline $\begin{array}{c}\text { Nombre } \\
\text { variable } \mathrm{ACM}\end{array}$ & Municipio & \# EAH & Segmento de mercado \\
\hline \multirow{3}{*}{$\begin{array}{c}\text { Zona } \\
\text { Zipa_Cogua y } \\
\text { Nemocón } \\
\text { (1) }\end{array}$} & Zipaquirá & 15 & \multirow{3}{*}{ Corporativo / Turístico } \\
\hline & Cogua & 2 & \\
\hline & Nemocón & 1 & \\
\hline \multirow{2}{*}{$\begin{array}{l}\text { Zona Toca y } \\
\text { Gacha } \\
2\end{array}$} & Tocancipá & 8 & \multirow{2}{*}{$\begin{array}{c}\text { Corporativo/ } \\
\text { Transportadores/ } \\
\text { Peregrinos religiosos }\end{array}$} \\
\hline & Gachancipá & 3 & \\
\hline $\begin{array}{c}\text { Sopó } \\
3\end{array}$ & Sopó & 7 & Corporativo / Turístico \\
\hline \multirow{2}{*}{$\begin{array}{l}\text { Zona Chía y } \\
\text { Cajicá } \\
\text { (4) }\end{array}$} & Chía & 8 & \multirow{2}{*}{$\begin{array}{c}\text { Corporativo/ } \\
\text { Universitario/ Turístico }\end{array}$} \\
\hline & Cajicá & 3 & \\
\hline \multirow{2}{*}{$\begin{array}{c}\text { Zona Cota y } \\
\text { Tenjo } \\
5\end{array}$} & Cota & 3 & \multirow[b]{2}{*}{ Corporativo } \\
\hline & Tenjo & 2 & \\
\hline $\begin{array}{c}\text { Tabio } \\
6\end{array}$ & Tabio & 3 & Rural/Turístico/Termales \\
\hline \multicolumn{2}{|c|}{ Total EAH investigados } & 55 & \\
\hline
\end{tabular}

Elaboración propia

Para el análisis de correspondencia múltiple, se consideraron 11 variables cualitativas (tabla 5) y cuatro variables cuantitativas (tabla 6) que se categorizaron para incluirlas en el ACM. Esta técnica resulta idónea para caracterizar la oferta de (EAH) presentes en los municipios de la provincia de Sabana Centro a partir de los factores estratégicos internos. 
Tabla 5. Variables cualitativas seleccionadas para el análisis ACM

\begin{tabular}{|c|c|c|}
\hline Variables cualitativas & Categoría & Convención \\
\hline $\begin{array}{l}\text { Nivel de formación del } \\
\text { administrador del EAH }\end{array}$ & $\begin{array}{l}\text { Básica: Primaria, bachillerato, U. Incompleta } \\
\text { Técnica: Técnica, tecnológica } \\
\text { Superior: Universitaria, posgradual }\end{array}$ & $\begin{array}{l}\text { Form_Técnica } \\
\text { Form_Superior }\end{array}$ \\
\hline Personería jurídica & $\begin{array}{l}\text { Persona natural } \\
\text { Sociedad empresarial }\end{array}$ & $\begin{array}{l}\text { Pers_Natural } \\
\text { Sociedad }\end{array}$ \\
\hline Misión, visión y valores & Existencia de misión, visión, valores & Mis_Vis_Val $(*)$ \\
\hline Políticas de recursos humanos & Existencia de políticas de recursos humanos & Pol_RH $(*)$ \\
\hline Página web & Existencia de página web del EAH & Web $(*)$ \\
\hline Servicios de alimentación & $\begin{array}{l}\text { Existencia de restaurante, bar, room service y/o } \\
\text { salones de eventos }\end{array}$ & AyB $(*)$ \\
\hline $\begin{array}{l}\text { Mecanismos para conocer al } \\
\text { cliente }\end{array}$ & $\begin{array}{l}\text { Existencia de mecanismos para conocer al } \\
\text { cliente (encuestas, estudios, otros) }\end{array}$ & Enc_cl $(*)$ \\
\hline Plan de ventas & Existencia de un plan de mercadeo y ventas & Plan_ventas_mdeo $(*)$ \\
\hline Protocolos de servicio & $\begin{array}{l}\text { Existencia de protocolos de servicio } \\
\text { documentados en el EAH }\end{array}$ & Prot_Serv $(*)$ \\
\hline $\begin{array}{l}\text { Mantenimiento a la } \\
\text { infraestructura }\end{array}$ & $\begin{array}{l}\text { Existencia de planes de mantenimiento a la } \\
\text { infraestructura del EAH }\end{array}$ & Mantto $\left({ }^{*}\right)$ \\
\hline Presencia en redes sociales & $\begin{array}{l}\text { Existencia de presencia en redes sociales } \\
\text { (Instagram, Facebook, Google, otras) con } \\
\text { información sobre servicios y actividades del } \\
\text { EAH }\end{array}$ & R_Soc $(*)$ \\
\hline Otros servicios & $\begin{array}{l}\text { Existencia de servicios complementarios al de } \\
\text { alojamiento (estacionamiento, gimnasio, } \\
\text { lavandería, otros) }\end{array}$ & Otros_serv $(*)$ \\
\hline \multirow{2}{*}{\multicolumn{3}{|c|}{$\begin{array}{l}\left({ }^{*}\right) \text { En la figura } 9 \text { se presentan los resultados del análisis ACM utilizando las convenciones descritas en esta tak } \\
\text { las preposiciones con y sin, para indicar que se cuenta o no con la presencia de las respectivas variables de an } \\
\text { propia. } \\
\text { Tabla } 6 \text {. Variables cuantitativas seleccionadas para el análisis ACM }\end{array}$}} \\
\hline & & \\
\hline Variables cuantitativas & Categoría & Convención \\
\hline $\begin{array}{l}\text { Tiempo de operación desde la } \\
\text { apertura }\end{array}$ & $\begin{array}{l}\text { Operación nueva: } 1 \text { a } 5 \text { años } \\
\text { Operación reciente: } 5 \text { a } 7 \text { años } \\
\text { Operación decenal: } 7 \text { a } 11 \text { años } \\
\text { Operación antigua: Mayor a } 11 \text { años }\end{array}$ & $\begin{array}{l}\text { Op_Nueva } \\
\text { Op_Reciente } \\
\text { Op_Decenal } \\
\text { Op_Antigüa }\end{array}$ \\
\hline Rango de tarifas por noche & $\begin{array}{l}\text { Económico: } \$ 30.000 \text { a } \$ 90.000 \\
\text { Medio: } \$ 91.000 \text { a } \$ 140.000 \\
\text { Premium: } \$ 141.000 \text { a } \$ 270.00\end{array}$ & 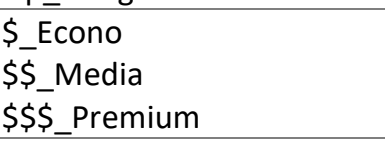 \\
\hline $\begin{array}{l}\text { Tamaño del EAH en número de } \\
\text { habitaciones }\end{array}$ & $\begin{array}{l}\text { Pequeño: } 2 \text { a } 19 \text { habitaciones } \\
\text { Mediano: } 20 \text { a } 39 \text { habitaciones } \\
\text { Grande: } 64 \text { a } 128 \text { habitaciones }\end{array}$ & $\begin{array}{l}\text { Hoteles_Pequeños } \\
\text { Hoteles_Medianos } \\
\text { Hoteles_Grandes }\end{array}$ \\
\hline $\begin{array}{l}\text { Calificación en redes de } \\
\text { viajeros (promedio ponderado } \\
\text { entre \# de comentarios y } \\
\text { calificación en tripadvisor.com } \\
\text { y booking.com) }\end{array}$ & $\begin{array}{l}\text { Nulo: } 0 \text { a } 5.9 \\
\text { Promedio: } 6 \text { a } 8 \\
\text { Superior: } 8.1 \text { a } 10\end{array}$ & $\begin{array}{l}\text { Rango_Cal_Nulo } \\
\text { Rango_Cal_Promedio } \\
\text { Rango_Cal_Superior }\end{array}$ \\
\hline
\end{tabular}

\section{Elaboración propia}

A partir de la información recopilada en la investigación y teniendo en cuenta los parámetros anteriores se realizó con el apoyo del software $R$ la gráfica de ACM que se presenta 
en la figura 9. Se encontraron dos grandes perfiles de EAH, los modernos definidos por los atributos ubicados en los cuadrantes II y III, y los EAH tradicionales en la parte derecha de la figura, en los cuadrantes I y IV. Los EAH tradicionales se ubican principalmente en las zonas 1 (Zipa, Cogua y Nemocón) y 2 (Toca y Gacha). En contraste, los modernos están presentes en las zonas 4 (Chía y Cajicá) y 5 (Cota y Tenjo).

Figura 7. Distribución de los atributos de los EAH resultado de análisis ACM

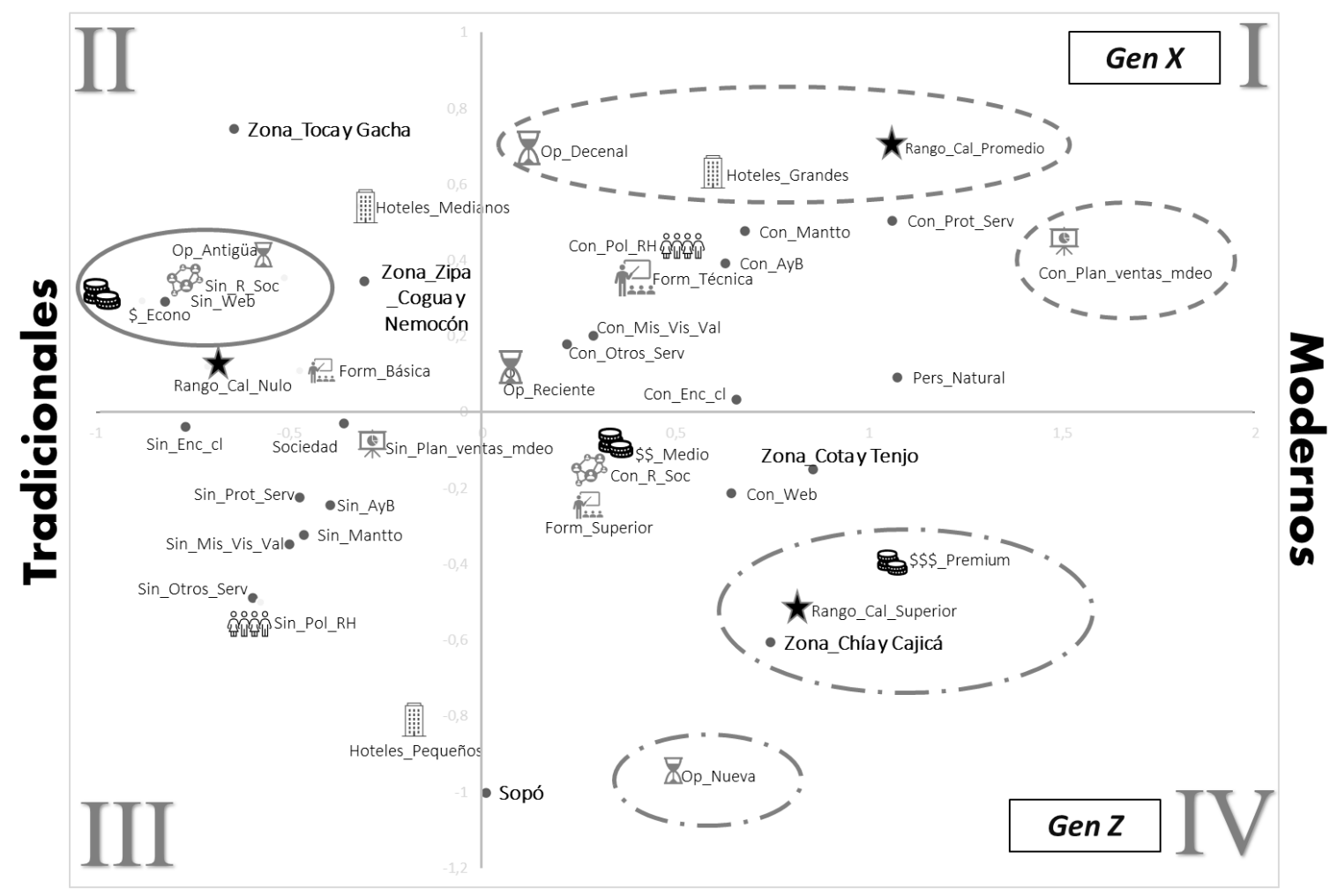

Elaboración propia

En el grupo los hoteles modernos, se identificaron dos subgrupos (figura 9): parte superior derecha que se denominó gen $X$ (cuadrante I) y parte inferior derecha que se denominó gen $Z$ (cuadrante IV). El perfil de los establecimientos de alojamiento y hospedaje modernos identificados como gen $X$ se asemejan a la generación adulta con presencia de desarrollo en los factores de estrategia. Se caracterizan por el atributo de "operación decenal" es decir operaciones con más de 7 años de funcionamiento, y "EAH grandes" debido a que contiene los EAH con mayor número de habitaciones de la provincia (más de 64 habitaciones). Esta categoría de EAH presentan la necesidad de ocupar el hotel para cubrir costos fijos y dar rendimiento a sus propietarios porque han organizado los esquemas de distribución y comunicación con el desarrollo de planes de mercadeo y ventas. Por otro lado, se interesan en el desarrollo de su talento humano a partir de la creación de políticas y de la formación soportada por el conocimiento desarrollado internamente que se preserva en protocolos de servicio documentados.

Los establecimientos de alojamiento y hospedaje modernos del cuadrante I, gen $X$, están dirigidos por administradores de formación técnica, la cual posiblemente les ha dado una base para dar importancia a elementos del direccionamiento estratégico como lo son la misión, visión y valores. Además, se caracterizan porque se han preocupado por ampliar la 
oferta del alojamiento con esquemas de servicios de alimentos y bebidas y otros servicios los cuales tienden a ser un atributo neutral para este perfil, pero el no tenerlo hace la diferencia como se evidencia en el perfil de EAH tradicional. Estos hoteles modernos son cuidadosos con el activo y el ambiente físico que presentan a sus huéspedes porque han estructurado planes de mantenimiento de infraestructura, equipos y mobiliario. Se evidencia también en este perfil que el rango de calificación en redes sociales para viajeros es promedio.

El siguiente perfil (cuadrante IV, parte inferior derecha), los denominados gen Z, es el grupo que ha crecido en medio del mundo digital, se caracterizan principalmente por el atributo "EAH de operación nueva" menor a 5 años, que cuentan con un mayor rango de calificaciones en redes de viajeros y una alta asociación a ofrecer tarifas premium. Los administradores tienen un nivel de educación superior y le han dado relevancia a la presencia en redes sociales y a contar con una página web.

Un tercer perfil, denominado establecimientos de alojamiento y hospedaje tradicionales, puede considerarse opuesto de los dos anteriores y es el que se encuentra en la parte izquierda del gráfico (cuadrantes II y III). Este perfil describe EAH que geográficamente corresponden a la zona 2 (Toca y Gacha) de tamaño mediano (20 a 39 habitaciones). Los atributos que más definen a los establecimientos de alojamiento y hospedaje tradicionales son: Operaciones de más de 11 años, que carecen de un sitio web, no están presentes en redes sociales y que no dan relevancia a la tenencia de políticas de talento humano. Este perfil de hoteles es administrado por personas con formación básica que no desarrollan elementos para dar un norte a su gestión como los son la misión, la visión y los valores. De igual forma no cuentan con políticas establecidas para el cuidado y mantenimientos de los activos y no buscan desarrollar ni proteger el conocimiento generado por la organización para formar a su equipo.

Se resalta el caso de Sopó, el cual se ubica en el eje vertical, pero con una ligera inclinación al cuadrante IV, que está conformado por EAH pequeños y cuyas características coinciden con los EAH modernos. Finalmente, el municipio de Tabio, con solo tres EAH y un segmento de clientes enfocado al turismo rural, no se incluyó en este análisis, dentro de las características de sus EAH se encuentran operaciones nuevas como decenales, estos son administrados por personas con formación superior que dieron relevancia a contar con página web y estar presentes en redes sociales, con lo cual han logrado calificaciones de nivel promedio y tarifas premium.

\section{CONCLUSIONES}

Las organizaciones en general y la vinculadas al sector turismo en particular buscan alcanzar una ventaja competitiva, al respeto García (2013) considera que la incorporación de intangibles enmarcados en temas de conocimiento, recursos organizativos, información e innovación y presencia en redes sociales son claves para impulsar la creación de valor, en los resultados del estudio se identificó la presencia de los tres factores estratégicos internos en los EAH de la provincia de Sabana Centro en diferentes proporciones, los hallazgos muestran brechas importantes que deben ser cerradas para que los mismos obtengan una mejor competitividad. Adicionalmente, para alcanzar un mejor desempeño debe existir un compromiso y voluntad política de los entes de la región para impulsar el tema del turismo. La búsqueda de información primaria muestra que los entes públicos como por ejemplo las secretarías de turismo municipal tienen diferentes grados de desarrollo y no se evidencia el 
desarrollo de herramientas para consolidar el destino y coordinar el trabajo de la oferta turística de la provincia. Además, tampoco se encontraron iniciativas gremiales que tengan representatividad ante los EAH y que los puedan apoyar para mejorar y articular sus negocios.

Al momento de analizar los factores propuestos por González-Rodríguez, et al (2018): activos organizacionales y activos reputacionales, y con el concepto de marketing mix (Bowie y Buttle, 2011) uno de los hallazgos más significativos dentro los resultados obtenidos revelan que los EAH en la provincia son gestionados por sus propietarios quienes cuenta con una baja formación y experiencia en turismo. Este hecho explica sus debilidades con relación a las competencias gerenciales, y en el desarrollo de procesos de marketing mix para lograr ventajas competitivas. Por esta razón, se considera que para lograr estas competencias es necesario fortalecer procesos de formación y de consultoría, en conjunto esto permitiría mejorar las habilidades gerenciales de los administradores de los EAH, mientras la consultoría brindaría un acompañamiento de un equipo interdisciplinario a través de planes de trabajo bien definido para que los EAH logren verdaderas ventajas competitivas.

Con relación a los activos reputacionales también considerados por GonzálezRodríguez, et al (2018) como elementos indispensables para mejorar el desempeño y la competitividad, los resultados de EAH de la provincia de Sabana Centro muestran debilidades que deben ser mejoradas para aprovechar y potenciar la belleza de sus paisajes y los atractivos turísticos, por lo cual se necesita desarrollar esfuerzos orientados a consolidar una oferta consistente y sostenible que optimice la competitividad de este sector.

Este trabajo propuso que los factores estratégicos internos en los EAH de la provincia de Sabana Centro que posibilitan una ventaja competitiva son: los activos organizacionales, el marketing mix y los activos reputacionales. Los resultados obtenidos evidencian relaciones entre los factores estratégicos internos y la obtención de mejores resultados, medidos en este caso por las calificaciones de redes sociales de viajeros o la posibilidad de cobrar tarifas superiores al promedio, siendo estas variables relevantes para hacer más rentables y competitivas estas organizaciones.

No se evidencia una diferenciación clara entre los establecimientos presentes en la región que se enfoque en atraer más segmentos de huéspedes y genere mayor fidelidad entre los mismos. A pesar de lo anterior, se encontraron dos perfiles de EAH presentes en la provincia, los modernos y los tradicionales: En la primera clasificación se encuentran los EAH ubicados en los municipios de Cota, Tenjo, Chía y Cajicá, en el perfil de EAH modernos se observaron dos subgrupos que tienen variables comunes y mayor presencia de factores estratégicos. El perfil de los EAH tradicionales es opuesto a los anteriores y describe EAH que geográficamente están ubicados en la zona Tocancipá, Gachancipá, Zipaquirá, Cogua y Nemocón, y evidencian poco desarrollo de los factores estratégicos internos.

La presente investigación fue desarrollada antes de la pandemia de la COVID-19 y se terminó de escribir durante el confinamiento preventivo obligatorio, periodo en el cual los indicadores están en caída y no son alentadores para el sector hotelero (Shapoval, et al, 2020; Herédia-Colaço y Rodrigues, 2021). Esta situación inesperada ha evidenciado la falta de preparación de los empresarios y la relevancia de los resultados encontrados en este estudio. Cabe destacar que este es un momento histórico clave para crear planes de recuperación enfocados al turismo. Urge preparar a los EAH de la provincia para su reapertura, las capacidades de sus administradores deben fortalecerse para un comienzo de operación bajo 
la nueva realidad de la industria y es imperativa la orientación para la obtención de soporte financiero para la recuperación.

Finalmente como líneas futuras de investigación se proponen: (a) desarrollar un análisis de pertinencia y priorización para generar acciones de mejora concretas para los perfiles de hoteles modernos y tradicionales, (b) evaluar la situación actual de los EAH analizados en el presente estudio para plantear alternativas de recuperación, de cara a afrontar las nuevas realidades que deberá asumir el sector después de superada la pandemia por la COVID-19, (c) identificar las necesidades y expectativas de los clientes para proponer a los EAH nuevos esquemas de operación enfocados a nichos específicos de mercado como por ejemplo el turismo de naturaleza y rural, turismo gastronómico, esquemas de sostenibilidad turística, mayor uso de tecnologías, sellos de bioseguridad, entre otros, (d) analizar las ventajas de la articulación público-privada para generar iniciativas que fortalezcan el destino turístico y (e) realizar estudios comparativos de este estudio en otras regiones del departamento o en otras regiones del país.

\section{REFERENCIAS BIBLIOGRÁFICAS}

Amit, R., y Schoemaker, P. J. . H. . (1993). Strategic Assets and Organizational Rent. Strategic Management Journal, 14(1), 33-46. https://doi.org/10.1002/smj.4250140105

Bačík, R., Fedorko, R., Abbas, E. W., Rigelský, M., Ivanková, V., y Obšatníková, K. (2019). The impact of selected quality management attributes on the profitability of top hotels in the visegrad group countries. Polish Journal of Management Studies, 19(1), 46-58. https://doi.org/10.17512/pims.2019.19.1.04

Bowie, D., y Buttle, F. (2011). Hospitality marketing : principles and practice / David Bowie y Francis Buttle. Boston: Routledge. Recuperado de http://unisabana22.gsl.com.mx:80/F?func=service\&doc library=CNA01\&local base $=$ CNA01\&doc number $=000178448 \&$ sequence $=000001 \&$ line number $=0001 \&$ func cod e=DB RECORDS\&service type=MEDIA

Brathwaite, R. (1992). Value-Chain Assessment of the Travel Experience. Cornell Hotel and Restaurant Administration Quarterly, 33, 5, 41-49. https://doi.org/10.1177/001088049203300525

Cámara de Comercio de Bogotá. (2005). Caracterización de las cadenas productivas de manufactura y servicios en Bogotá y Cundinamarca. Recuperado de: https://bibliotecadigital.ccb.org.co/bitstream/handle/11520/2886/6232006 411 11619 Caracterizacion de las cadenas productivas DEF.pdf? sequence=1\&isAllo $\underline{\text { wed }=\mathrm{y}}$

Camisón, C., Puig-Denia. A., Forés, B., Fabra, M., Muñóz, A. y Muñóz, C. (2015). The Importance of Internal Resources and Capabilities and Destination Resources to Explain Firm Competitive Position in the Spanish Tourism Industry. International Journal of Tourism Research, 18, 341-356. https://doi.org/10.1002/jtr.2053

Consejo Regional de Competitividad (2010). Plan Regional de Competitividad Bogotá y Cundinamarca 2010-2019. Recuperado de http://www.sdp.gov.co/sites/default/files/pa002-3planregionalcompetitividad.pdf 
Díaz, L., y Morales, M. (2012). Análisis Estadístico de datos multivariados. (U. N. de Colombia, Ed.) (Primera). Bogotá, Colombia.

Ducci, A., y Mertens, L. (1997). Formación basada en competencia laboral : situación actuMertens, L. (1997). Formación basada en competencia laboral : situación actual y perspectivas Herramientas para la trans ormación. Seminario Internacional sobre Formación Basada en Competencia Labora. Seminario Internacional Sobre Formación Basada En Competencia Laboral: Situación Actual y Perspectivas, 1, Guanajuato, México, 1996. Documentos Presentados. Montevideo: Cinterfor, 1997.

Enríquez-De-La-O, J. F. (2015). Resource-based view and dynamic capabilities - Achieving competitive advantage through internal resources and competences. Vezetéstudomány / Budapest Management Review, 46(11), 50-61.

Foroudi, P. (2020). Corporate brand strategy: Drivers and outcomes of hotel industry's brand orientation. International Journal of Hospitality Management, 88, 102519. https://doi.org/10.1016/j.ijhm.2020.102519

García de León, S. (2013). Importancia estratégica de los activos intangibles en la industria hotelera. Hospitalidad-ESDAI, Enero-Juni, 7-25.

Gobernación de Cundinamarca (2016). Plan de Desarrollo Cundinamarca 2016-2020. Gobernación de Cundinamarca Recuperado de http://www.cundinamarca.gov.co/wcm/connect/2a9dd7d1-d693-414a-94cd-

37fe5f901e7d/PLAN+DE+DESARROLLO+VERSION+FINAL.pdf?MOD=AJPERES\&CVID=ID IW39U

Gobernación de Cundinamarca (2020). Plan de Desarrollo Cundinamarca 2020-2024. Gobernación de Cundinamarca. Recuperado de http://www.cundinamarca.gov.co/wcm/connect/37b90ffc-f445-462b-8faa8a16f4427fe8/PLAN+DE+DESARROLLO+PLIEGOS1 compressed.pdf?MOD=AJPERES\&C VID=njCfayi\&CVID=njCfayi\&CVID=njCfayi\&CVID=njCfayi\&CVID=njCfayi

González-Rodríguez, M. R., Jiménez-Caballero, J. L., Martín-Samper, R. C., Köseoglu, M. A., y Okumus, F. (2018). Revisiting the link between business strategy and performance: Evidence from hotels. International Journal of Hospitality Management, 72 https://doi.org/10.1016/j.ijhm.2017.11.008

Gottschalg, O., y Zollo, M. (2007). Interest alignment and competitive advantage https://doi.org/10.5465/amr.2007.24351356

Gursoy, D., y Swanger, N. (2007). Performance-enhancing internal strategic factors and competencies: Impacts on financial success. International Journal of Hospitality Management, 26(1), 213-227. https://doi.org/10.1016/i.ijhm.2006.01.004

Guzzo, R. A., y Dickson, M. W. (1996). Teams in Organizations: Recent Research on Performance and Effectiveness. Annual Review of Psychology, 47(1), 307-338. https://doi.org/10.1146/annurev.psych.47.1.307

Herédia-Colaço, V., y Rodrigues, H. (2021). Hosting in turbulent times: Hoteliers' perceptions and strategies to recover from the Covid-19 pandemic. International Journal of Hospitality Management, 94, 102835. https://doi.org/10.1016/j.ijhm.2020.102835 
Köseoglu, M. A., Altin, M., Chan, E., \& Aladag, O. F. (2020). What are the key success factors for strategy formulation and implementation? Perspectives of managers in the hotel industry. International Journal of Hospitality Management, 89, 102574. https://doi.org/10.1016/j.ijhm.2020.102574

Langvinienè, N., y Daunoravičiūtè, I. (2015). Factors Influencing the Success of Business Model in the Hospitality Service Industry. Procedia - Social and Behavioral Sciences, 213, 902910. https://doi.org/10.1016/i.sbspro.2015.11.503

Millán, C. H., y Gómez, M. del R. (2018). Factores e indicadores de competitividad hotelera. Compendium, 21(40).

MinCIT-CITUR (2019). MinCIT-Citur | Estadísticas. Recuperado de http://citur.gov.co/estadisticas/df prestadores historico/all/41

MinCIT-CITUR (2021). Establecimientos de alojamiento y hospedaje con RNT. Recuperado de http://www.citur.gov.co/estadisticas/departamental\#gsc.tab=0 (C)

MinCIT (2009) Norma técnica NTSH sectorial colombiana 006. Recuperado de https://fontur.com.co/aym document/aym normatividad/2009/NTSH006-09.pdf

MinCIT (2018). Plan Sectorial De Turismo 2018-2022. Bogotá, Colombia: Ministerio de Comercio, Industria y Turismo de Colombia. Recuperado de http://www.mincit.gov.co/loader.php?IServicio=Documentos\&IFuncion=verPdf\&id=8 5331\&name=PST revision consulta publica.pdf\&prefijo=file

Porter, M. E. (1996). What is strategy. Harvard Business Review, November, 61-78.

Pozo, A., Campos, J., Sánchez, J., y Lara, M. (2011, January). Capital humano y salarios en la hostelería española : Un análisis regional. Revista de Estudios Regionales No.91, 7195.

Rubio, A., y Aragón, A. (2008). Recursos estratégicos en la pymes. Revista Europea de Dirección y Economía de La Empresa, 17(1), 103-126.

Rubio, Á., Jiménez, I., y Mercado, C. (2017). Reputación corporativa online en la hotelería: el caso TripAdvisor. Esic Market Economics and Business Journal, 48(3), 595-608. https://doi.org/10.7200/esicm.158.0483.4e

Rukstad, M. G., y Collis, D. (2008). Can You Say What Your Strategy Is? Harvard Business Review, (April), 82-91.

Sabana Centro Cómo Vamos (2016). Sabana Centro, Una Provincia Sostenible. Recuperado de http://sabanacentrocomovamos.org/home/wp-content/uploads/2018/10/SABANACENTRO.-Una-provincia-sostenible.pdf

Sampieri, R. H., y Torres, C. P. M. (2018). Metodología de la investigación Las rutas cuantitativa, cualitativa y mixta. (McGraw-Hill Interamericana, Ed.). Recuperado de https://www-ebooks7-24-com.ez.unisabana.edu.co/?il=6443

Sánchez, M., Fernández, M., y Mier-Terán, J. (2020). El uso y la importancia de las redes sociales en el sector hotelero desde la perspectiva de los responsables de su gestión. Investigaciones Turísticas, 20, 50-78. DOI. https://doi.org/10.14198/INTURI2020.20.03 
Scheel, A. (2010). Evaluación operacional y financiera aplicada a la industria hotelera (3rd ed.). Bogotá, Colombia: Universidad Externado de Colombia.

Shapoval, V., Hägglund, P., Pizam, A., Abraham, V., Carlbäck, M., Nygren, T. y Madison, R. (2020). The COVID-19 pandemic effects on the hospitality industry using social systems theory: A multi-country comparison. International Journal of Hospitality Management, 94, 102813. https://doi.org/10.1016/i.ijhm.2020.102813

Varvaressos, S. (2018). Framework for Tourism Industries: Tourism System and Tourism Value Chain. The Emerald Handbook of Entrepreneurship in Tourism, Travel and Hospitality, 19-31. https://doi.org/10.1108/978-1-78743-529-220181002

Wikipedia (2015). Mapa de la provincia Sabana Centro, Colombia. Recuperado de https://commons.wikimedia.org/wiki/File:Colombia - Cundinamarca Sabana Centro.svg

Wernerlfet, B. (1984). A Resource-based View of the Firm. Strategic Management Journal, 5, 171-180. https://doi.org/10.1177/1056492611436225

\section{CONTRIBUCIÓN DE LOS AUTORES:}

Autor 1: Recopilación y análisis de datos.

Autor 2: Revisión crítica de los contenidos, la adaptación del trabajo como artículo, así como la revisión y actualización de datos, conclusiones y la versión final de documento para su publicación.

Autor 3: Revisión crítica de los contenidos, la adaptación del trabajo como artículo, así como la revisión y actualización de datos, conclusiones y la versión final de documento para su publicación.

\section{AGRADECIMIENTOS:}

Los tres autores agradecen a los propietarios y gerentes de los hoteles que participaron en la realización de la investigación, también a aquellos que con sus comentarios permitieron perfeccionar el documento hasta lograr su publicación. 